\title{
Hörmander Class of Pseudo-Differential Operators on Compact Lie Groups and Global Hypoellipticity
}

\author{
Michael Ruzhansky • Ville Turunen · Jens Wirth
}

Received: 15 April 2013 / Revised: 21 November 2013 / Published online: 8 April 2014

(C) Springer Science+Business Media New York 2014

\begin{abstract}
In this paper we give several global characterisations of the Hörmander class $\Psi^{m}(G)$ of pseudo-differential operators on compact Lie groups in terms of the representation theory of the group. The result is applied to give criteria for the ellipticity and the global hypoellipticity of pseudo-differential operators in terms of their matrix-valued full symbols. Several examples of the first and second order globally hypoelliptic differential operators are given, in particular of operators that are locally not invertible nor hypoelliptic but globally are. Where the global hypoelliptiticy fails, one can construct explicit examples based on the analysis of the global symbols.
\end{abstract}

Keywords Pseudo-differential operators · compact Lie groups · microlocal analysis · elliptic operators $\cdot$ global hypoellipticity $\cdot$ Leibniz formula

Communicated by Hartmut Führ.

The first author was supported by the EPSRC Leadership Fellowship EP/G007233/1. The third author was supported by the EPSRC grant EP/E062873/1.

M. Ruzhansky ( $\bowtie)$

Department of Mathematics, Imperial College London, 180 Queen's Gate, London SW7 2AZ, UK

e-mail: m.ruzhansky@imperial.ac.uk

V. Turunen

Department of Mathematics and Systems Analysis, Aalto University,

P.O. Box 11100, 00076 Aalto, Finland

e-mail: ville.turunen@aalto.fi

J. Wirth

Institut für Analysis, Dynamik und Modellierung, Universität Stuttgart, Pfaffenwaldring 57,

70569 Stuttgart, Germany

e-mail: jens.wirth@iadm.uni-stuttgart.de 
Mathematics Subject Classification Primary $35 \mathrm{~S} 05 \cdot$ Secondary 22E30

\section{Introduction}

For a smooth closed manifold $M$ we denote by $\Psi^{m}(M)$ the set of Hörmander's pseudodifferential operators on $M$, i.e., the class of operators which in all local coordinate charts give operators in $\Psi^{m}\left(\mathbb{R}^{n}\right)$. Operators in $\Psi^{m}\left(\mathbb{R}^{n}\right)$ are characterised by the symbols satisfying

$$
\left|\partial_{\xi}^{\alpha} \partial_{x}^{\beta} a(x, \xi)\right| \leq C(1+|\xi|)^{m-|\alpha|}
$$

for all multi-indices $\alpha, \beta$ and all $x, \xi \in \mathbb{R}^{n}$. An operator in $\Psi^{m}(M)$ is called elliptic if all of its localisations are locally elliptic. The principal symbol of a pseudo-differential operator on $M$ can be invariantly defined as function on the cotangent bundle $T^{*} M$, but it is not possible to control lower order terms in the same way. If one fixes a connection, however, it is possible to make sense of a full symbol, see e.g. Widom [23].

However, on a Lie group $G$ it is a very natural idea to use the Fourier analysis and the global Fourier transform on the group to analyse the behaviour of operators which are originally defined by their localisations. This allows one to make a full use of the representation theory and of many results available from the harmonic analysis on groups. In [12] and [10], the first two authors carried out a comprehensive noncommutative analysis of an analogue of the Kohn-Nirenberg quantization on $\mathbb{R}^{n}$, in terms of the representation theory of the group. This yields a full matrix-valued symbol defined on the product $G \times \widehat{G}$, which can be viewed as a non-commutative version of the phase space. The calculus and other properties of this quantization resemble those well-known in the local theory on $\mathbb{R}^{n}$. However, due to the non-commutativity of the group in general, the full symbol is matrix-valued, with the dimension of the matrix symbol $a(x, \xi)$ at $x \in G$ and $[\xi] \in \widehat{G}$ equal to the dimension of the representation $\xi$. This construction is inspired by that of Taylor [20] but is carried out entirely in terms of the group $G$ without referring to its Lie algebra and the Euclidean pseudo-differential classes there. It is parallel to the Kohn-Nirenberg quantization on $\mathbb{R}^{n}$ ([9]), see formula (2). In [2] (see also [21]), pseudo-differential operators have been characterised by their commutator properties with the vector fields. In [10], we gave a different version of this characterisation relying on the commutator properties in Sobolev spaces, which led in [12] (see also [10]) to characterisations of symbols of operators in $\Psi^{m}(G)$. However, the results there still rely on commutator properties of operators and some of them are taken as assumptions. One aim of this paper is to eliminate such assumptions from the characterisation, and to give its different versions relying on different choices of difference operators on $\widehat{G}$. This will be given in Theorem 2.2 .

In Theorem 2.6 we give another simple description of Hörmander's classes on the group $\mathrm{SU}(2)$ and on the 3 -sphere $\mathbb{S}^{3}$. This is possible due to the explicit analysis carried out in [12] and the explicit knowledge of the representation of SU(2). We note that this approach works globally on the whole sphere, e.g. compared to the analysis of Sherman [16] working only on the hemisphere (see also [17]). 
We give two applications of the characterisation provided by Theorem 2.2. First, we characterise elliptic operators in $\Psi^{m}(G)$ by their matrix-valued symbols. Similar to the toroidal case (see [1]) this can be applied further to spectral problems. For example, this characterisation will be instrumental in establishing a version of the Gohberg lemma and estimates for the essential spectrum for operators on compact Lie groups, see [5].

Second, we give a sufficient condition for the global hypoellipticity of pseudodifferential operators. Since the hypoellipticity depends on the lower order terms of an operator, a knowledge of the full symbol becomes crucial. Here, we note that while classes of hypoelliptic symbols can be invariantly defined on manifolds by localisation (see, e.g., Shubin [18]), the lower order terms of symbols can not. From this point of view conditions of Theorem 5.1 appear natural as they refer to the full symbol defined globally on $G \times \widehat{G}$.

The global hypoellipticity of vector fields and second order differential operators on the torus has been extensively studied in the literature, see e.g. [3], [6], and references therein. In this paper we study the global hypoellipticity problem for pseudodifferential operators on general compact Lie groups, and discuss a number of explicit examples on the group $\mathbb{S}^{3} \cong \mathrm{SU}(2)$. We say that an operator $A$ is globally hypoelliptic on $G$ if $A u=f$ and $f \in C^{\infty}(G)$ imply $u \in C^{\infty}(G)$. Thus, we give examples of first and second order differential operators which are not locally hypoelliptic and are not covered by Hörmander's sum of the squares theorem but which can be seen to be globally hypoelliptic by the techniques described in this paper. For example, if $X$ is a normalised left-invariant vector field on $\mathbb{S}^{3}$ and $\partial_{X}$ is the derivative with respect to $X$, then

the operator $\partial_{X}+c$ is globally hypoelliptic if and only if ic $\notin \frac{1}{2} \mathbb{Z}$.

We note that this operator is not locally hypoelliptic, but the knowledge of the global symbol allows us to draw conclusions about its global properties. It is interesting to observe that the number theory plays a role in the global properties, in some way similar to the appearance of the Liouville numbers in the global properties of vector fields on the torus. For example, the failure of the D'Alembertian $D_{3}^{2}-D_{1}^{2}-D_{2}^{2}$ to be globally hypoelliptic can be related to properties of the triangular numbers, and an explicit counterexample to the global hypoellipticity can be consequently constructed based on them. We also look at the cases of the sub-Laplacian and other operators, which are covered by Hörmander's theorem, but for which we construct an explicit parametrix which turns out to be a pseudo-differential operator with the global matrixvalued symbol of type $\left(\frac{1}{2}, 0\right)$. Such examples can also be easily extended to variable coefficient versions.

Another application of Theorem 2.2 to obtain the sharp Gårding inequality on compact Lie groups appears in [13]. Moreover, in [15] it will play a crucial role in establishing $L^{p}$-multiplier theorems on general compact Lie groups, the result which was also announced in [14].

We will now introduce some notation. Let throughout this paper $G$ be a compact Lie group of (real) dimension $\operatorname{dim} G$ with the unit element $e$, and denote by $\widehat{G}$ the set 
of all equivalence classes of continuous irreducible unitary representations of $G$. For necessary details on Lie groups and their representations we refer to [10], but recall some basic facts for the convenience of the reader and in order to fix the notation. Each $[\xi] \in \widehat{G}$ corresponds to a homomorphism $\xi: G \rightarrow \mathrm{U}\left(d_{\xi}\right)$ with $\xi(x y)=\xi(x) \xi(y)$ satisfying the irreducibility condition $\mathbb{C}^{d_{\xi}}=\operatorname{span}\{\xi(x) v: x \in G\}$ for any given $v \in \mathbb{C}^{d_{\xi}} \backslash\{0\}$. The number $d_{\xi}$ is referred to as the dimension of the representation $\xi$.

We always understand the group $G$ as a manifold endowed with the (normalised) bi-invariant Riemannian structure. Of major interest for us is the following version of the Peter-Weyl theorem defining the group Fourier transform $\mathcal{F}: L^{2}(G) \rightarrow \ell^{2}(\widehat{G})$.

Theorem 1.1 The space $L^{2}(G)$ decomposes as the orthogonal direct sum of biinvariant subspaces parameterised by $\widehat{G}$,

$$
L^{2}(G)=\bigoplus_{[\xi] \in \widehat{G}} \mathcal{H}^{\xi}, \quad \mathcal{H}^{\xi}=\left\{x \mapsto \operatorname{Tr}(A \xi(x)): A \in \mathbb{C}^{d_{\xi} \times d_{\xi}}\right\}
$$

the decomposition given by the Fourier series

$$
f(x)=\sum_{[\xi] \in \widehat{G}} d_{\xi} \operatorname{Tr}(\xi(x) \widehat{f}(\xi)), \quad \widehat{f}(\xi)=\int_{G} f(x) \xi(x)^{*} \mathrm{~d} x .
$$

Furthermore, the following Parseval identity is valid,

$$
\|f\|_{L^{2}(G)}^{2}=\sum_{[\xi] \in \widehat{G}} d \xi\|\widehat{f}(\xi)\|_{H S}^{2}=:\|\widehat{f}\|_{\ell^{2}(\widehat{G})^{2}} .
$$

The notion of Fourier series extends naturally to $C^{\infty}(G)$ and to the space of distributions $\mathcal{D}^{\prime}(G)$ with convergence in the respective topologies.

Now, any operator $A$ on $G$ mapping $C^{\infty}(G)$ to $\mathcal{D}^{\prime}(G)$ gives rise to a matrix-valued full symbol $\sigma_{A}(x, \xi) \in \mathbb{C}^{d_{\xi}} \times d_{\xi}$ defined by

$$
\sigma_{A}(x, \xi):=\xi(x)^{*}(A \xi)(x)
$$

which can be understood either pointwise or distributionally, as the product of a smooth matrix-valued function $\xi(x)^{*}$ with the matrix-valued distribution $A \xi$, i.e. $\sigma_{A}(\cdot, \xi)=$ $\xi^{*} A \xi$ as a distribution in the first variable, for all $[\xi] \in \widehat{G}$. Then it can be shown that

$$
A f(x)=\sum_{[\xi] \in \widehat{G}} d_{\xi} \operatorname{Tr}\left(\xi(x) \sigma_{A}(x, \xi) \widehat{f}(\xi)\right)
$$

holds as $\mathcal{D}^{\prime}$-convergent series. If it happens that the operator $A$ maps $C^{\infty}(G)$ to itself, then (2) holds in the strong topology of $C^{\infty}(G)$. For $A$ and $\sigma_{A}$ related by (2) we also write $A=\mathrm{Op}\left(\sigma_{A}\right)$. 
For a rather comprehensive treatment of this quantization we refer to [10] and [12]. We denote the right-convolution kernel of $A$ by $R_{A}$, so that

$$
A f(x)=\int_{G} K_{A}(x, y) f(y) \mathrm{d} y=\int_{G} R_{A}\left(x, y^{-1} x\right) f(y) \mathrm{d} y,
$$

where $\mathrm{d} y$ is the normalised Haar measure on $G$. The symbol $\sigma_{A}$ and the rightconvolution kernel $R_{A}$ are related by $\sigma_{A}(x, \xi)=\int_{G} R_{A}(x, y) \xi(y)^{*} \mathrm{~d} y$.

The first aim of this paper is to characterise Hörmander's class of pseudo-differential operators $\Psi^{m}(G)$ by the behaviour of their full symbols, with applications to the ellipticity and global hypoellipticity of operators.

The plan of this paper is as follows. In the next section we give several equivalent symbolic characterisations of pseudo-differential operators by their symbols. These characterisations are based on difference operators acting on sequences of matrices $\widehat{G} \ni[\xi] \mapsto \sigma(\xi) \in \mathbb{C}^{d_{\xi} \times d_{\xi}}$ of varying dimension. We also give an application to pseudo-differential operators on the group SU(2). Section 3 contains the proof and some useful lemmata on difference operators. Section 4 contains the criteria for the ellipticity of operators in terms of their full matrix-valued symbols as well as a finite version of the Leibniz formula for difference operators associated to the group representations. Finally, Sect. 5 contains criteria for the global hypoellipticity of pseudodifferential operators and a number of examples.

\section{Characterisations of Hörmander's Class}

The following statement is based on difference operators. They are defined as follows. We say that $Q_{\xi}$ is a difference operator of order $k$ if it is given by

$$
Q_{\xi} \widehat{f}(\xi)=\widehat{q_{Q} f}(\xi)
$$

for a function $q=q_{Q} \in C^{\infty}(G)$ vanishing of order $k$ at the identity $e \in G$, i.e., $\left(P_{x} q_{Q}\right)(e)=0$ for all left-invariant differential operators $P_{x} \in \operatorname{Diff}^{k-1}(G)$ of order $k-1$. We denote the set of all difference operators of order $k$ as $\operatorname{diff}^{k}(\widehat{G})$.

In the sequel, for a given function $q \in C^{\infty}(G)$ it will be also convenient to denote the associated difference operator, acting on Fourier coefficients, by

$$
\Delta_{q} \widehat{f}(\xi):=\widehat{q f}(\xi)
$$

Definition 2.1 A collection of $n$ first order difference operators $\triangle_{1}, \ldots, \triangle_{n} \in$ $\operatorname{diff}^{1}(\widehat{G})$ is called admissible, if the corresponding functions $q_{1}, \ldots, q_{n} \in C^{\infty}(G)$ satisfy $\mathrm{d} q_{j}(e) \neq 0, j=1, \ldots, n$, and $\operatorname{rank}\left(\mathrm{d} q_{1}(e), \ldots, \mathrm{d} q_{n}(e)\right)=\operatorname{dim} G$. It follows, in particular, that $e$ is an isolated common zero of the family $\left\{q_{j}\right\}_{j=1}^{n}$. An admissible 
collection is called strongly admissible if

$$
\bigcap_{j=1}^{n}\left\{x \in G: q_{j}(x)=0\right\}=\{e\}
$$

For a given admissible selection of difference operators on a compact Lie group $G$ we use multi-index notation

$$
\triangle_{\xi}^{\alpha}:=\triangle_{1}^{\alpha_{1}} \cdots \triangle_{n}^{\alpha_{n}} \text { and } q^{\alpha}(x):=q_{1}(x)^{\alpha_{1}} \cdots q_{n}(x)^{\alpha_{n}} .
$$

Furthermore, there exist corresponding differential operators $\partial_{x}^{(\alpha)} \in \operatorname{Diff}^{|\alpha|}(G)$ such that Taylor's formula

$$
f(x)=\sum_{|\alpha| \leq N-1} \frac{1}{\alpha !} q^{\alpha}\left(x^{-1}\right) \partial_{x}^{(\alpha)} f(e)+\mathcal{O}\left(h(x)^{N}\right), \quad h(x) \rightarrow 0
$$

holds true for any smooth function $f \in C^{\infty}(G)$ and with $h(x)$ the geodesic distance from $x$ to the identity element $e$. An explicit construction of operators $\partial_{x}^{(\alpha)}$ in terms of $q^{\alpha}(x)$ can be found in [10, Section 10.6]. In addition to these differential operators $\partial_{x}^{(\alpha)} \in \operatorname{Diff}^{|\alpha|}(G)$ we introduce operators $\partial_{x}^{\alpha}$ as follows. Let $\partial_{x_{j}} \in \operatorname{Diff}^{1}(G), 1 \leq j \leq$ $\operatorname{dim} G$, be a collection of left-invariant first order differential operators corresponding to some linearly independent family of the left-invariant vector fields on $G$. We denote $\partial_{x}^{\alpha}=\partial_{x_{1}}^{\alpha_{1}} \partial_{x_{2}}^{\alpha_{2}} \cdots$. We note that in most estimates we can freely replace operators $\partial_{x}^{(\alpha)}$ by $\partial_{x}^{\alpha}$ and the other way around since they can be clearly expressed in terms of each other.

After fixing the notion of difference operators, we have to specify orders. Each of the bi-invariant subspaces $\mathcal{H}^{\xi}$ of Theorem 1.1 is an eigenspace of the Laplacian $\mathcal{L}$ on $G$ with the corresponding eigenvalue $-\lambda_{\xi}^{2}$. Based on these eigenvalues we define $\langle\xi\rangle=\left(1+\lambda_{\xi}^{2}\right)^{1 / 2}$. In particular we recover the familiar characterisation

$$
f \in H^{s}(G) \quad \Longleftrightarrow \quad\langle\xi\rangle^{s} \widehat{f}(\xi) \in \ell^{2}(\widehat{G}) .
$$

We are now in a position to formulate the main result of this paper.

Theorem 2.2 Let A be a linear continuous operator from $C^{\infty}(G)$ to $\mathcal{D}^{\prime}(G)$. Then the following statements are equivalent:

(A) $A \in \Psi^{m}(G)$.

(B) For every left-invariant differential operator $P_{x} \in \operatorname{Diff}^{k}(G)$ of order $k$ and every difference operator $Q_{\xi} \in \operatorname{diff}^{\ell}(\widehat{G})$ of order $\ell$ the symbol estimate

$$
\left\|Q_{\xi} P_{x} \sigma_{A}(x, \xi)\right\|_{o p} \leq C_{Q_{\xi} P_{x}}\langle\xi\rangle^{m-\ell}
$$

is valid. 
(C) For an admissible selection $\triangle_{1}, \ldots, \triangle_{n} \in \operatorname{diff}^{1}(\widehat{G})$ we have

$$
\left\|\triangle_{\xi}^{\alpha} \partial_{x}^{\beta} \sigma_{A}(x, \xi)\right\|_{o p} \leq C_{\alpha \beta}\langle\xi\rangle^{m-|\alpha|}
$$

for all multi-indices $\alpha, \beta$. Moreover, $\operatorname{sing} \operatorname{supp} R_{A}(x, \cdot) \subseteq\{e\}$.

(D) For a strongly admissible selection $\triangle_{1}, \ldots, \triangle_{n} \in \operatorname{diff}^{1}(\widehat{G})$ we have

$$
\left\|\triangle_{\xi}^{\alpha} \partial_{x}^{\beta} \sigma_{A}(x, \xi)\right\|_{o p} \leq C_{\alpha \beta}\langle\xi\rangle^{m-|\alpha|}
$$

for all multi-indices $\alpha, \beta$.

It follows from the proof that if conditions (C) or (D) are satisfied for one admissible (strongly admissible, resp.) selection of first order differences, they are automatically satisfied for any admissible (strongly admissible, resp.) selection of first order differences. The set of symbols $\sigma_{A}$ satisfying either of equivalent conditions $(B)-(D)$ will be denoted by $\mathscr{S}^{m}(G)$.

Among other things, this theorem removes the assumption of the conjugation invariance from the list of conditions used in [10]. For $u \in G$, denote $A_{u} f:=A(f \circ \phi) \circ \phi^{-1}$, where $\phi(x)=x u$. It can be shown that

$$
R_{A_{u}}(x, y)=R_{A}\left(x u^{-1}, u y u^{-1}\right) \text { and } \sigma_{A_{u}}(x, \xi)=\xi(u)^{*} \sigma_{A}\left(x u^{-1}, \xi\right) \xi(u) .
$$

On one hand, we have $A \in \Psi^{m}(G)$ if and only if $A_{u} \in \Psi^{m}(G)$ for all $u \in G$. On the other hand, as a corollary of Theorem 2.2 this can be expressed in terms of difference operators, showing that the difference conditions are conjugation invariant. In fact, to draw such a conclusion, we even do not need to know that conditions $(B)-(D)$ characterise the class $\Psi^{m}(G)$ :

Corollary 2.3 Let the symbol $\sigma_{A}$ of a linear continuous operator from $C^{\infty}(G)$ to $\mathcal{D}^{\prime}(G)$ satisfy either of the assumptions $(B),(C),(D)$ of Theorem 2.2. Then the symbols $\sigma_{A_{u}}$ satisfy the same symbolic estimates for all $u \in G$.

This statement follows immediately from the condition $(B)$ if we observe that the difference operators applied to the symbol $\sigma_{A_{u}}$ just lead to a different set of difference operators in the symbolic inequalities. Alternatively, one can observe that the change of variables by $\phi$ amounts to the change of the basis in the representation spaces of $\widehat{G}$, thus leaving the condition $(B)$ invariant again.

Example 2.4 On the torus $\mathbb{T}^{n}=\mathbb{R}^{n} / \mathbb{Z}^{n}$, the family of functions $q_{j}(x)=\mathrm{e}^{-2 \pi \mathrm{i} x_{j}}-1$, $j=1, \ldots, n$, gives rise to a strongly admissible collection of difference operators

$$
\triangle_{j} a(\xi)=\triangle_{q_{j}} a(\xi)=a\left(\xi+e_{j}\right)-a(\xi), \quad j=1, \ldots, n,
$$

with $\xi \in \widehat{\mathbb{T}^{n}} \simeq \mathbb{Z}^{n}$, where $e_{j}$ is the $j^{t h}$ unit vector in $\mathbb{Z}^{n}$. As an immediate consequence of Theorem 2.2 we recover the fact that the class $\Psi^{m}\left(\mathbb{T}^{n}\right)$ can be characterised by the difference conditions on their toroidal symbols:

$$
\left|\triangle_{\xi}^{\alpha} \partial_{x}^{\beta} \sigma_{A}(x, \xi)\right| \leq C_{\alpha \beta}(1+|\xi|)^{m-|\alpha|}
$$


uniformly for all $x \in \mathbb{T}^{n}$ and $\xi \in \mathbb{Z}^{n}$. We refer to [11] for details of the corresponding toroidal quantization of operators on $\mathbb{T}^{n}$. See also [1].

In Theorem 2.2 we characterise Hörmander operators entirely by symbol assumptions based on a set of admissible difference operators. There does not seem to be a canonic choice for difference operators, for different applications different selections of them seem to be most appropriate. We will comment on some of them.

(1) Simplicity. Difference operators have a simple structure, if the corresponding functions are just matrix coefficients of irreducible representations. Then application of difference operators at fixed $\xi$ involves only matrix entries from finitely many (neighbouring) representations.

(2) Taylor's formula. Any admissible selection of first order differences allows for a Taylor series expansion.

(3) Leibniz rule. Leibniz rules have a particularly simple structure if one chooses difference operators of a certain form, see Proposition 4.3. Moreover, operators of this form yield a strongly admissible collection, see Lemma 4.4.

Example 2.5 We will conclude this section with an application for the particular group SU(2). Certain explicit calculations on SU(2) have been done in [12] and the background on the necessary representation theory of SU(2) can be also found in [10]. Representations on SU(2) are parametrised by half-integers $\ell \in \frac{1}{2} \mathbb{N}_{0}$ (so-called quantum numbers) and are of dimension $d_{\ell}=2 \ell+1$. Here $\mathbb{N}_{0}=\mathbb{N} \cup\{0\}$. Thus, irreducible representations of SU(2) are given by matrices $t^{\ell}(x) \in \mathbb{C}^{(2 \ell+1) \times(2 \ell+1)}, \ell \in \frac{1}{2} \mathbb{N}_{0}$, after some choice of the basis in the representation spaces. One admissible selection of difference operators corresponds to functions $q_{-}, q_{+}, q_{0} \in C^{\infty}(\mathrm{SU}(2))$ defined by

$$
q_{-}=t_{-1 / 2,+1 / 2}^{1 / 2}, \quad q_{+}=t_{+1 / 2,-1 / 2}^{1 / 2}, \quad q_{0}=t_{-1 / 2,-1 / 2}^{1 / 2}-t_{+1 / 2,+1 / 2}^{1 / 2},
$$

where we denote

$$
t^{1 / 2}=\left(\begin{array}{ll}
t_{-1 / 2,-1 / 2}^{1 / 2} & t_{-1 / 2,+1 / 2}^{1 / 2} \\
t_{+1 / 2,-1 / 2}^{1 / 2} & t_{+1 / 2,+1 / 2}^{1 / 2}
\end{array}\right)
$$

Our analysis on SU(2) is in fact equivalent to the corresponding analysis on the 3sphere $\mathbb{S}^{3} \cong \mathrm{SU}(2)$, the isomorphism given by the identification of $\mathrm{SU}(2)$ with $\mathbb{S}^{3} \subset \mathbb{H}$ in the quaternion space $\mathbb{H}$, with the quaternionic product on $\mathbb{S}^{3}$ corresponding to the matrix multiplication on $\mathrm{SU}(2)$. Writing explicitly an isomorphism $\Phi: \mathbb{S}^{3} \rightarrow \mathrm{SU}(2)$ as

$$
\left(x_{0}, x_{1}, x_{2}, x_{3}\right)=x \mapsto \Phi(x)=\left(\begin{array}{cc}
x_{0}+\mathrm{i} x_{3} & x_{1}+\mathrm{i} x_{2} \\
-x_{1}+\mathrm{i} x_{2} & x_{0}-\mathrm{i} x_{3}
\end{array}\right),
$$

the identity matrix $e \in \mathrm{SU}(2)$ corresponds to the vector $\mathbf{1}$ in the basis decomposition $x=\left(x_{m}\right)_{m=0}^{3} \mapsto x_{0} \mathbf{1}+x_{1} \mathbf{i}+x_{2} \mathbf{j}+x_{3} \mathbf{k}$ on $\mathbb{H}$. We note that the family $\triangle_{+}:=\triangle_{q_{+}}$, $\triangle_{-}:=\triangle_{q_{-}}$and $\triangle_{0}:=\triangle_{q_{0}}$ is not strongly admissible because in addition to $e \in \mathrm{SU}(2)$ (or to $\mathbf{1} \in \mathbb{H}$ ) they have another common zero at $-e$ (or at $\mathbf{- 1}$ ).

Following [10] and [12], we simplify the notation on $\mathrm{SU}(2)$ and $\mathbb{S}^{3}$ by writing $\sigma_{A}(x, \ell)$ for $\sigma_{A}\left(x, t^{\ell}\right), \ell \in \frac{1}{2} \mathbb{N}_{0}$, and we refer to these works for the explicit formulae 
for the difference operators $\Delta_{+}, \Delta_{-}, \Delta_{0}$. We denote $\Delta_{\ell}^{\alpha}=\triangle_{+}^{\alpha_{1}} \triangle_{-}^{\alpha_{2}} \triangle_{0}^{\alpha_{3}}$. In [12] we proved that the operator and the entrywise supremum norms are uniformly equivalent for symbols of pseudo-differential operators from $\Psi^{m}(\mathrm{SU}(2))$. As a corollary to Theorem 2.2, Corollary 2.3, and [10, Theorem 12.4.3] we get

Theorem 2.6 Let $A$ be a linear continuous operator from $C^{\infty}(\mathrm{SU}(2))$ to $\mathcal{D}^{\prime}(\mathrm{SU}(2))$. Then $A \in \Psi^{m}(\mathrm{SU}(2))$ if and only if sing supp $R_{A}(x, \cdot) \subset\{e\}$ and

$$
\sup _{i j}\left|\triangle_{\ell}^{\alpha} \partial_{x}^{\beta} \sigma_{A}(x, \ell)_{i j}\right| \leq C_{\alpha \beta}(1+\ell)^{m-|\alpha|}
$$

for all multi-indices $\alpha, \beta \in \mathbb{N}_{0}^{3}$ and all $l \in \frac{1}{2} \mathbb{N}_{0}$. Moreover, in these cases we also have the rapid off-diagonal decay property of symbols, namely, we have

$$
\left|\triangle_{\ell}^{\alpha} \partial_{x}^{\beta} \sigma_{A}(x, \ell)_{i j}\right| \leq C_{A \alpha \beta m N}(1+|i-j|)^{-N}(1+\ell)^{m-|\alpha|}
$$

uniformly in $x \in \mathrm{SU}(2)$, for every $N \geq 0$, all $\ell \in \frac{1}{2} \mathbb{N}_{0}$, every multi-indices $\alpha, \beta \in \mathbb{N}_{0}^{3}$, and for all matrix column/row numbers $i, j$.

We note that Theorem 2.6 holds in exactly the same way if we replace SU(2) by $\mathbb{S}^{3}$. Moreover, according to Theorem 1.1 the statement of Theorem 2.6 holds without the kernel condition sing supp $R_{A}(x, \cdot) \subset\{e\}$ provided that instead of the admissible family $\Delta_{+}, \Delta_{-}, \Delta_{0}$ we take a strongly admissible family of difference operators in $\operatorname{diff}^{1}(\widehat{\mathrm{SU}(2)})$, for example one given by four first order difference operators corresponding to functions $q_{-}, q_{+}, t_{-1 / 2,-1 / 2}^{1 / 2}-1$ and $t_{+1 / 2,+1 / 2}^{1 / 2}-1$.

\section{Proof of Theorem 2.2}

\section{$3.1(A) \Longrightarrow(C)$}

By [10, Thm. 10.9.6] (and in the notation used there) we know that condition (A) is equivalent to $\sigma_{A} \in \Sigma^{m}(G)=\bigcap_{k} \Sigma_{k}^{m}(G)$, where $\Sigma_{0}^{m}(G)$ already corresponds to assumption (C). There is nothing to prove.

\section{$3.2(C) \Longrightarrow(D)$}

Evident.

Also the following partial converse is true. If (D) is satisfied, then the symbol estimates imply for the corresponding right-convolution kernel $R_{A}(x, \cdot)$ of $A$ that

$$
\operatorname{sing} \operatorname{supp} R_{A}(x, \cdot) \subseteq \bigcap_{j=1}^{n}\left\{y \in G: q_{j}(y)=0\right\}=\{e\} .
$$




\section{$3.3(D) \Longrightarrow(B)$}

For a given strongly admissible selection of first order differences, we can apply Taylor's formula to the function $q_{Q}$ corresponding to the difference operator $Q \in$ $\operatorname{diff}^{\ell}(\widehat{G})$. Hence, we obtain

Lemma 3.1 Let $Q \in \operatorname{diff}^{\ell}(\widehat{G})$ be an arbitrary difference operator of order $\ell$. Then

$$
Q=\sum_{\ell \leq|\alpha| \leq N-1} c_{\alpha} \triangle_{\xi}^{\alpha}+Q_{N}
$$

for suitable constants $c_{\alpha} \in \mathbb{C}$ and a difference operator $Q_{N} \in \operatorname{diff}^{N}(\widehat{G})$.

Consequently, (D) together with (B) for differences of order larger than $N$ implies (B). More precisely, with the notation $\|\sigma(x, \xi)\|_{C^{\infty}}$ for 'any' $C^{\infty}$-seminorm of the form $\sup _{x}\left\|P_{x} \sigma(x, \cdot)\right\|_{o p}$ and

$$
\mathscr{R}_{N}^{m}=\left\{\sigma_{A}:\left\|Q_{\xi} \sigma_{A}(x, \xi)\right\|_{C^{\infty}} \lesssim\langle\xi\rangle^{m-\ell} \quad \forall \ell>N \forall Q_{\xi} \in \operatorname{diff}^{\ell}(\widehat{G})\right\}
$$

and

$$
\mathscr{S}_{\ell}^{m}=\left\{\sigma_{A}:\left\|\triangle_{\xi}^{\alpha} \sigma_{A}(x, \xi)\right\|_{C^{\infty}} \lesssim\langle\xi\rangle^{m-\ell} \quad \forall|\alpha|=\ell\right\}
$$

we obtain

$$
\mathscr{R}_{0}^{m}=\mathscr{S}_{0}^{m} \cap \mathscr{S}_{1}^{m} \cap \cdots \cap \mathscr{S}_{N}^{m} \cap \mathscr{R}_{N}^{m}
$$

while (B) corresponds to taking all symbols from $\mathscr{R}_{0}^{m}$. This implies

$$
\mathscr{R}_{0}^{m}=\left(\bigcap_{\ell \geq 0} \mathscr{S}_{\ell}^{m}\right) \cap \mathscr{R}_{\infty}^{m}
$$

with

$$
\mathscr{R}_{\infty}^{m}=\left\{\sigma_{A}:\left\|Q_{\xi} \sigma(x, \xi)\right\|_{C^{\infty}} \lesssim C_{N}\langle\xi\rangle^{-N} \quad \forall N \forall Q_{\xi} \in \operatorname{diff}{ }^{\infty}(\widehat{G})\right\}
$$

Assumption (D) corresponds to the first intersection $\mathscr{S}^{m}=\bigcap_{\ell} \mathscr{S}_{\ell}^{m}$.

It remains to show that $\mathscr{S}^{m} \subset \mathscr{R}_{\infty}^{m}$, which is done in the sequel.

Let $Q_{\xi} \in \operatorname{diff}^{\infty}(\widehat{G})$, i.e. $q_{Q} \in C^{\infty}(G)$ is vanishing to infinite order at $e$. Let further

$$
p(y):=\sum_{j=1}^{n} q_{j}^{2}(y) .
$$

Then $p(y) \neq 0$ for $y \neq e$ and $p$ vanishes of the second order at $y=e$. Hence, $q_{Q}(y) /|p(y)|^{N} \in C^{\infty}(G)$ for arbitrary $N$ and, therefore, 


$$
q_{Q}(y) R_{A}(x, y)=\frac{q_{Q}(y)}{|p(y)|^{N}}|p(y)|^{N} R_{A}(x, y)
$$

can be estimated in terms of symbol bounds for $\sigma_{A} \in \mathscr{S}^{m}$. Indeed, by Lemma 3.2 it follows that $|p(y)|^{N} R_{A}(x, y) \in H^{-m-c+2 N}(G \times G)$. Letting $N \rightarrow \infty$ proves the claim.

Lemma 3.2 Let $\sigma_{A} \in \mathscr{S}^{m}$. Then $R_{A}(x, \cdot) \in H^{-m-c}(G)$ for all $c>\frac{1}{2} \operatorname{dim} G$.

Proof This just follows from $\left\|\sigma_{A}(x, \xi)\right\|_{H S} \leq \sqrt{d \xi}\left\|\sigma_{A}(x, \xi)\right\|_{o p}$ in combination with the observation that

$$
\sum_{[\xi]} d_{\xi}^{2}\langle\xi\rangle^{-2 c}<\infty
$$

for $c>\frac{1}{2} \operatorname{dim} G$ (which is equivalent to $H^{c}(G) \hookrightarrow L^{2}(G)$ being Hilbert-Schmidt).

$$
3.4(B) \Longleftrightarrow(C) \Longleftrightarrow(D)
$$

Evident from the above.

$$
3.5(B) \Longrightarrow(A)
$$

We use the commutator characterisation of the class $\Psi^{m}(G)$, [10, Thm. 10.7.7]. It says that it is sufficient to show that

(1) any symbol $\sigma_{A}$ satisfying condition (B) gives rise to a bounded linear operator $A: H^{m}(G) \rightarrow L^{2}(G)$

(2) the symbol $\sigma_{[X, A]}$ of the commutator of $A$ and any left-invariant vector field $X$ also satisfies (B).

The nuclearity of $C^{\infty}(G)$ allows one to split the first statement into the corresponding results for purely $x$ - and purely $\xi$-dependent symbols; both situations are evident. As for the second statement, we use the symbolic calculus established in [10, Thm. 10.7.9] for classes of symbols including, in particular, the class $\mathscr{S}^{m}$, which writes the symbol of the composition as the asymptotic sum

$$
\sigma_{[X, A]} \sim \sigma_{X} \sigma_{A}-\sigma_{A} \sigma_{X}+\sum_{|\alpha|>1} \frac{1}{\alpha !}\left(\triangle_{\xi}^{\alpha} \sigma_{X}\right)\left(\partial_{x}^{(\alpha)} \sigma_{A}\right)
$$

The first term $\sigma_{X} \sigma_{A}-\sigma_{A} \sigma_{X}$ is on the level of right-convolution kernels $R_{A}(x, \cdot)$ given by

$$
\left(X_{y}-X_{y}^{R}\right) R_{A}(x, y)
$$

where $X^{R}$ is the right-invariant vector field tangent to $X$ at $e$ (and the index $y$ describes the action as a differential operator with respect to the $y$-variable). Apparently, $X_{y}-X_{y}^{R}$ is a smooth vector field on $G$ and can thus be written as 


$$
\sum_{j=1}^{n} q_{j}(y) \partial_{j}^{R}
$$

with $q_{j}(e)=0$ and $\partial_{j}^{R}$ suitable right-invariant derivatives. Hence, on the symbolic level $\sigma_{X} \sigma_{A}-\sigma_{A} \sigma_{X}=\sum_{j=1}^{n} \triangle_{j}\left(\sigma_{A} \sigma_{\partial_{j}}\right)$ with $\triangle_{j} \in \operatorname{diff}^{1}(\widehat{G})$, defined by $\triangle_{j} \widehat{f}(\xi)=$ $\widehat{q_{j} f}(\xi)$. Now the statement follows from Lemma 3.4 by the aid of the asymptotic Leibniz rule [10, Thm. 10.7.12]:

Lemma 3.3 Let $\triangle_{1}, \ldots, \triangle_{n} \in \operatorname{diff}^{1}(\widehat{G})$ be a set of admissible differences. For any $Q_{\xi} \in \operatorname{diff}^{\ell}(\widehat{G})$ there exist $Q_{\xi, \alpha} \in \operatorname{diff}^{\ell}(\widehat{G})$ such that

$$
Q_{\xi}\left(\sigma_{1} \sigma_{2}\right) \sim\left(Q_{\xi} \sigma_{1}\right) \sigma_{2}+\sum_{|\alpha|>0}\left(Q_{\xi, \alpha} \sigma_{1}\right)\left(\triangle_{\xi}^{\alpha} \sigma_{2}\right)
$$

for any (fixed) set of admissible differences.

Lemma 3.4 Let $A$ be a differential operator of order $m$. Then $\sigma_{A} \in \mathscr{S}^{m}$.

Proof Lemma 3.4 is a consequence of the already proved implications $(A) \Longrightarrow$ $(C) \Longrightarrow(D) \Longrightarrow(B)$. Explicit formulae for the difference operators applied to symbols of differential operators can be also found in [10, Prop. 10.7.4] and provide an elementary proof of this statement.

\section{Ellipticity and Leibniz Formula}

As an application of Theorem 2.2 we will give a characterisation of the elliptic operators in $\Psi^{m}(G)$ in terms of their global symbols.

Theorem 4.1 An operator $A \in \Psi^{m}(G)$ is elliptic if and only if its matrix valued symbol $\sigma_{A}(x, \xi)$ is invertible for all but finitely many $[\xi] \in \widehat{G}$, and for all such $\xi$ satisfies

$$
\left\|\sigma_{A}(x, \xi)^{-1}\right\|_{o p} \leq C\langle\xi\rangle^{-m}
$$

for all $x \in G$.

Thus, both statements are equivalent to the existence of $B \in \Psi^{-m}(G)$ such that $I-B A$ and $I-A B$ are smoothing. For the ellipticity condition (4) on the general matrix level it is not enough to assume that $\left|\operatorname{det} \sigma_{A}(x, \xi)\right|^{1 / d_{\xi}} \geq C\langle\xi\rangle^{m}$ due to the in general growing dimension of the matrices. However, if we assume that the smallest singular value of the matrix $\sigma_{A}(x, \xi)$ is greater or equal than $C\langle\xi\rangle^{m}$ uniformly in $x$ and (all but finitely many) $\xi$, then condition (4) follows.

Let the collection $q_{1}, \ldots, q_{n}$ give an admissible collection of difference operators and let $\partial_{x}^{(\gamma)}$ be the corresponding family of differential operators as in the Taylor expansion formula (3). As an immediate corollary of Theorem 4.1 and [10, Thm. 10.9.10] we get 
Corollary 4.2 Let $A \in \Psi^{m}(G)$ be elliptic. Moreover, assume that $\sigma_{A}(x, \xi) \sim$ $\sum_{j=0}^{\infty} \sigma_{A_{j}}(x, \xi)$, where $\operatorname{Op}\left(\sigma_{A_{j}}\right) \in \Psi^{m-j}(G)$. Let $\sigma_{B}(x, \xi) \sim \sum_{k=0}^{\infty} \sigma_{B_{k}}(x, \xi)$, where $\sigma_{B_{0}}(x, \xi)=\sigma_{A_{0}}(x, \xi)^{-1}$ for large $\xi$, and the symbols $\sigma_{B_{k}}$ are defined recursively by

$$
\sigma_{B_{N}}(x, \xi)=-\sigma_{B_{0}}(x, \xi) \sum_{k=0}^{N-1} \sum_{j=0}^{N-k} \sum_{|\gamma|=N-j-k} \frac{1}{\gamma !}\left[\triangle_{\xi}^{\gamma} \sigma_{B_{k}}(x, \xi)\right] \partial_{x}^{(\gamma)} \sigma_{A_{j}}(x, \xi) .
$$

Then $\mathrm{Op}\left(\sigma_{B_{k}}\right) \in \Psi^{-m-k}(G), B=\mathrm{Op}\left(\sigma_{B}\right) \in \Psi^{-m}(G)$, and the operators $A B-I$ and $B A-I$ are in $\Psi^{-\infty}(G)$.

First, we give some preliminary results. In Lemma 3.3 we gave an asymptotic Leibniz formula but here we will present its finite version. Given a continuous unitary matrix representation $\eta=\left[\eta_{i j}\right]_{1 \leq i, j \leq \ell}: G \rightarrow \mathbb{C}^{\ell \times \ell}, \ell=d_{\eta}$, let $q(x)=\eta(x)-I$ (i.e. $q_{i j}=\eta_{i j}-\delta_{i j}$ with Kronecker's deltas $\delta_{i j}$ ), and define

$$
\mathbb{D}_{i j} \widehat{f}(\xi):=\widehat{q_{i j} f}(\xi)
$$

In the previous notation, we can write $\mathbb{D}_{i j}=\Delta_{q_{i j}}$. However, here, to emphasize that $q_{i j}$ 's correspond to the same representation, and to distinguish with difference operators with a single subindex, we will use the notation $\mathbb{D}$ instead. For a multi-index $\gamma \in \mathbb{N}_{0}^{\ell^{2}}$, we will write $|\gamma|=\sum_{i, j=1}^{\ell}\left|\gamma_{i j}\right|$, and for higher order difference operators we write $\mathbb{D}^{\gamma}=\mathbb{D}_{11}^{\gamma_{11}} \mathbb{D}_{12}^{\gamma_{12}} \cdots \mathbb{D}_{\ell, \ell-1}^{\gamma_{\ell, \ell-1}} \mathbb{D}_{\ell \ell}^{\gamma_{\ell \ell}}$.

For simplicity, we will abbreviate writing $a=a(\xi)$ and $b=b(\xi)$. Let $k \in \mathbb{N}$ and let $\alpha, \beta \in \mathbb{N}^{k}$ be such that $1 \leq \alpha_{j}, \beta_{j} \leq \ell$ for all $j \in\{1, \cdots, k\}$. Let us define a grand kth order difference $\overrightarrow{\mathbb{D}}^{k}$ by

$$
\left(\overrightarrow{\mathbb{D}}^{k} a\right)_{\alpha \beta}=\overrightarrow{\mathbb{D}}_{\alpha \beta}^{k} a:=\mathbb{D}_{\alpha_{1} \beta_{1}} \cdots \mathbb{D}_{\alpha_{k} \beta_{k}} a
$$

where we may note that the first order differences $\mathbb{D}_{\alpha_{j} \beta_{j}}$ commute with each other. We may compute with "matrices"

$$
\overrightarrow{\mathbb{D}}^{k} a=\left[\overrightarrow{\mathbb{D}}_{\alpha \beta}^{k} a\right]_{\alpha, \beta \in\{1, \cdots, \ell\}^{k}}
$$

using the natural operations, e.g.

$$
\left(\left(\overrightarrow{\mathbb{D}}^{k} a\right)\left(\overrightarrow{\mathbb{D}}^{k} b\right)\right)_{\alpha \beta}:=\sum_{\gamma \in\{1, \cdots, \ell\}^{k}}\left(\overrightarrow{\mathbb{D}}_{\alpha \gamma}^{k} a\right)\left(\overrightarrow{\mathbb{D}}_{\gamma \beta}^{k} b\right)
$$

In contrast to Lemma 3.3 with an asymptotic Leibniz formula for arbitrary difference operators, operators $\overrightarrow{\mathbb{D}}^{k}$ satisfy the finite Leibniz formula: 
Proposition 4.3 For all $k \in \mathbb{N}$ and $\alpha, \beta \in\{1, \cdots, \ell\}^{k}$ we have

$$
\overrightarrow{\mathbb{D}}_{\alpha \beta}^{k}(a b)=\sum_{|\varepsilon|,|\delta| \leq k \leq|\varepsilon|+|\delta|} C_{\alpha \beta \varepsilon \delta}^{k}\left(\mathbb{D}^{\varepsilon} a\right)\left(\mathbb{D}^{\delta} b\right),
$$

with the summation taken over all $\varepsilon, \delta \in \mathbb{N}_{0}^{\ell^{2}}$ satisfying $|\varepsilon|,|\delta| \leq k \leq|\varepsilon|+|\delta|$. In particular, for $k=1$, we have

$$
\begin{aligned}
\mathbb{D}_{i j}(a b) & =\left(\mathbb{D}_{i j} a\right) b+a\left(\mathbb{D}_{i j} b\right)+\left(\left(\overrightarrow{\mathbb{D}}^{1} a\right)\left(\overrightarrow{\mathbb{D}}^{1} b\right)\right)_{i j} \\
& =\left(\mathbb{D}_{i j} a\right) b+a\left(\mathbb{D}_{i j} b\right)+\sum_{k=1}^{\ell}\left(\mathbb{D}_{i k} a\right)\left(\mathbb{D}_{k j} b\right) .
\end{aligned}
$$

Proof Let us first show the case of $k=1$ given in formula (6). Recalling the notation $q_{i j}=\xi_{i j}-\delta_{i j}$, from the unitarity of the representation $\xi$, for all $x, y \in G$, we readily obtain the identity

$$
q_{i j}(x)=q_{i j}\left(x y^{-1}\right)+q_{i j}(y)+\sum_{k=1}^{\ell} q_{i k}\left(x y^{-1}\right) q_{k j}(y) .
$$

If now $f, g \in C^{\infty}(G)$, we consequently get

$$
q_{i j} \cdot(f * g)=\left(q_{i j} f\right) * g+f *\left(q_{i j} g\right)+\sum_{k=1}^{\ell}\left(q_{i k} f\right) *\left(q_{k j} g\right),
$$

which on the Fourier transform side gives (6).

For $k \geq 2$, let us argue inductively. Taking differences is linear, so the main point is to analyse the application of a first order difference $\mathbb{D}_{\alpha_{1} \beta_{1}}$ to the term $\left(\left(\overrightarrow{\mathbb{D}}^{1} a\right)\left(\overrightarrow{\mathbb{D}}^{1} b\right)\right)_{\alpha_{2} \beta_{2}}$. By (6) we have

$$
\begin{aligned}
\mathbb{D}_{\alpha_{1} \beta_{1}}\left(\left(\overrightarrow{\mathbb{D}}^{1} a\right)\left(\overrightarrow{\mathbb{D}}^{1} b\right)\right)_{\alpha_{2} \beta_{2}}= & \left(\left(\overrightarrow{\mathbb{D}}^{1} \mathbb{D}_{\alpha_{1} \beta_{1}} a\right)\left(\overrightarrow{\mathbb{D}}^{1} b\right)\right)_{\alpha_{2} \beta_{2}}+\left(\left(\overrightarrow{\mathbb{D}}^{1} a\right)\left(\overrightarrow{\mathbb{D}}^{1} \mathbb{D}_{\alpha_{1} \beta_{1}} b\right)\right)_{\alpha_{2} \beta_{2}} \\
& +\left(\left(\overrightarrow{\mathbb{D}}^{2} a\right)\left(\overrightarrow{\mathbb{D}}^{2} b\right)\right)_{\alpha \beta},
\end{aligned}
$$

where $\alpha=\left(\alpha_{1}, \alpha_{2}\right)$ and $\beta=\left(\beta_{1}, \beta_{2}\right)$. Proceeding by induction, we notice that applying a first order difference to the term $\left(\left(\overrightarrow{\mathbb{D}}^{k} a\right)\left(\overrightarrow{\mathbb{D}}^{k} b\right)\right)_{\alpha \beta}$ would introduce a sum of old type terms plus the new term $\left(\left(\overrightarrow{\mathbb{D}}^{k+1} a\right)\left(\overrightarrow{\mathbb{D}}^{k+1} b\right)\right)_{\widetilde{\alpha} \widetilde{\beta}}$. Thus, a $k$ th order difference operator applied to the product symbol $a b$ produces a linear combination of terms of the form $\left(\mathbb{D}^{\varepsilon} a\right)\left(\mathbb{D}^{\delta} b\right)$, where $|\varepsilon|,|\delta| \leq k \leq|\varepsilon|+|\delta|$, completing the proof. 
Difference operators $\mathbb{D}$ associated to the representations are particularly useful in view of the finite Leibniz formula in Proposition 4.3 and the fact that the collection of all differences $\mathbb{D}_{i j}$ over all $\xi$ and $i, j$ is strongly admissible:

Lemma 4.4 The family of difference operators associated to the family of functions $\left\{q_{i j}=\xi_{i j}-\delta_{i j}\right\}_{[\xi] \in \widehat{G}, 1 \leq i, j \leq d_{\xi}}$ is strongly admissible. Moreover, this family has a finite subfamily associated to finitely many representations which is still strongly admissible.

Proof We observe that there exists a homomorphic embedding of $G$ into $U(N)$ for a large enough $N$ and this embedding itself is a representation of $G$ of dimension $N$. Decomposing this representation into irreducible components gives a finite collection of representations. The common zero set of the corresponding family $\left\{q_{i j}\right\}$ is $e$ which means that it is strongly admissible.

We note that on the group $\mathrm{SU}(2)$ or on $\mathbb{S}^{3}$, by this argument with $N=2$, or by the discussion in Sect. 2 the four function $q_{i j}$ corresponding to the representation $\left[t^{\ell}\right] \in \widehat{\mathrm{SU}(2)}$ with $\ell=\frac{1}{2}$ of dimension two, $d_{\ell}=2$, already give a strongly admissible collection of four difference operators.

We can now apply Proposition 4.3 to the question of inverting the symbols on $G \times \widehat{G}$. We formulate this for symbol classes with $(\rho, \delta)$ behaviour as this will be also used in Sect. 5.

Lemma 4.5 Let $m \geq m_{0}, 1 \geq \rho>\delta \geq 0$ and let us fix difference operators $\left\{\mathbb{D}_{i j}\right\}_{1 \leq i, j \leq d_{\xi_{0}}}$ corresponding to some representation $\xi_{0} \in \widehat{G}$. Let the matrix symbol $a=a(x, \xi)$ satisfy

$$
\left\|\mathbb{D}^{\gamma} \partial_{x}^{\beta} a(x, \xi)\right\|_{o p} \leq C_{\beta \gamma}\langle\xi\rangle^{m-\rho|\gamma|+\delta|\beta|}
$$

for all multi-indices $\beta, \gamma$. Assume also that $a(x, \xi)$ is invertible for all $x \in G$ and $[\xi] \in \widehat{G}$, and satisfies

$$
\left\|a(x, \xi)^{-1}\right\|_{o p} \leq C\langle\xi\rangle^{-m_{0}}
$$

for all $x \in G$ and $[\xi] \in \widehat{G}$, and if $m_{0} \neq m$ in addition that

$$
\left\|a(x, \xi)^{-1}\left[\mathbb{D}^{\gamma} \partial_{x}^{\beta} a(x, \xi)\right]\right\|_{o p} \leq C\langle\xi\rangle^{-\rho|\gamma|+\delta|\beta|}
$$

for all $x \in G$ and $[\xi] \in \widehat{G}$. Then the matrix symbol $a^{-1}$ defined by $a^{-1}(x, \xi)=$ $a(x, \xi)^{-1}$ satisfies

$$
\left\|\mathbb{D}^{\gamma} \partial_{x}^{\beta} a^{-1}(x, \xi)\right\|_{o p} \leq C_{\beta \gamma}\langle\xi\rangle^{-m_{0}-\rho|\gamma|+\delta|\beta|}
$$

for all multi-indices $\beta, \gamma$.

Proof Let us denote $b(x, \xi):=a(x, \xi)^{-1}$ and estimate $\partial_{x}^{\beta} b$ first. Suppose we have proved that

$$
\left\|\partial_{x}^{\beta} b(x, \xi)\right\|_{o p} \leq C_{\beta}\langle\xi\rangle^{-m_{0}+\delta|\beta|}
$$


whenever $|\beta| \leq k$. We proceed by induction. Let us study the order $k+1$ cases $\partial_{x_{j}} \partial_{x}^{\beta} b$ with $|\beta|=k$. Since $a(x, \xi) b(x, \xi)=I$, by the usual Leibniz formula we get

$$
a \partial_{x_{j}} \partial_{x}^{\beta} b=-\sum_{\beta_{1}+\beta_{2}=\beta+e_{j},\left|\beta_{2}\right| \leq|\beta|} C_{\beta_{1} \beta_{2}}\left(\partial_{x}^{\beta_{1}} a\right)\left(\partial_{x}^{\beta_{2}} b\right) .
$$

From (8), (9), (10) and (12) we obtain the desired estimate

$$
\left\|\partial_{x_{j}} \partial_{x}^{\beta} b(x, \xi)\right\|_{o p} \leq C_{\beta}\langle\xi\rangle^{-m_{0}+\delta|\beta|+\delta}
$$

Let us now estimate $\mathbb{D}^{\gamma} b$. The argument is more complicated than that for the $\partial_{x}$ derivatives because the Leibniz formula in Proposition 4.3 has more terms. Indeed, the Leibniz formula in Proposition 4.3 applied to $a(x, \xi) b(x, \xi)=I$ gives

$$
\mathbb{D}_{i j} b+b\left(\mathbb{D}_{i j} a\right) b+\sum_{k=1}^{d_{\xi_{0}}} b\left(\mathbb{D}_{i k} a\right) \mathbb{D}_{k j} b=0 .
$$

Writing these equations for all $1 \leq i, j \leq d \xi_{0}$ gives a linear system on $\left\{\mathbb{D}_{i j} b\right\}_{i j}$ with coefficients of the form $I+b \mathbb{D}_{k l} a$ for suitable sets of indices $k, l$. Since $\left\|b \mathbb{D}_{k l} a\right\|_{o p} \leq$ $C\langle\xi\rangle^{-\rho}$, we can solve it for $\left\{\mathbb{D}_{i j} b\right\}_{i j}$ for large $\langle\xi\rangle$. The inverse of this system is bounded, and $\left\|b\left(\mathbb{D}_{i j} a\right) b\right\|_{o p} \leq C\langle\xi\rangle^{-m_{0}-\rho}$, implying that $\left\|\mathbb{D}_{i j} b\right\|_{o p} \leq C\langle\xi\rangle^{-m_{0}-\rho}$.

Suppose now we have proved that

$$
\left\|\mathbb{D}^{\gamma} b(x, \xi)\right\|_{o p} \leq C_{\gamma}\langle\xi\rangle^{-m_{0}-\rho|\gamma|}
$$

whenever $|\gamma| \leq k$. We proceed by induction. Let us study the order $k+1$ cases $\mathbb{D}_{i j} \mathbb{D}^{\gamma} b$ with $|\gamma|=k$. Now $a(x, \xi) b(x, \xi)=I$, so that

$$
\mathbb{D}_{i j} \mathbb{D}^{\gamma}(a b)=0
$$

where by Proposition 4.3 the right hand side is a sum of terms of the form $\left(\mathbb{D}^{\varepsilon} a\right)\left(\mathbb{D}^{\eta} b\right)$, where $|\varepsilon|,|\eta| \leq k+1 \leq|\varepsilon|+|\eta|$. Especially, we look at the terms $\mathbb{D}^{\varepsilon} a \mathbb{D}^{\eta} b$ with $|\eta|=k+1$, since the other terms can be estimated by $\langle\xi\rangle^{-\rho(k+1)}$ due to (13) and (8). Writing the linear system of equations on $\mathbb{D}_{i j} \mathbb{D}^{\eta^{\prime}} b$ produced by the Leibniz formula for all $i, j$ and all $\left|\eta^{\prime}\right|=k$, we see that the matrix coefficients in front of matrices $\mathbb{D}_{i j} \mathbb{D}^{\eta^{\prime}} b$ are sums of the terms of the form $\mathbb{D}^{\varepsilon} a$. The main term in each coefficient is $a(x, \xi)$ corresponding to $\varepsilon=0$, while the other terms corresponding to $\varepsilon \neq 0$ can be estimated by $\langle\xi\rangle^{m-\rho}$ in view of (13) and (8). It follows that we can solve $\mathbb{D}_{i j} \mathbb{D}^{\eta^{\prime}} b$ from this system and the solution matrix can be estimated by $\langle\xi\rangle^{-m_{0}}$ in view of (9) since its main term is $a(x, \xi)$. Therefore, all terms of the type $\mathbb{D}_{i j} \mathbb{D}^{\eta^{\prime}}$ can be estimated by

$$
\left\|\mathbb{D}_{i j} \mathbb{D}^{\eta^{\prime}} b(x, \xi)\right\|_{o p} \leq C_{\eta^{\prime}, i, j}\langle\xi\rangle^{-m_{0}-\rho(k+1)}
$$

which is what was required to prove. By combining these two arguments we obtain estimate (11). 
Proof of Theorem 4.1 Assume first that $A \in \Psi^{m}(G)$ is elliptic. Then it has a parametrix $B \in \Psi^{-m}(G)$ such that $A B-I, B A-I \in \Psi^{-\infty}(G)$. By the composition formulae for the matrix valued symbols in [10, Thm. 10.7.9] and Theorem 2.2 it follows that $\operatorname{Op}\left(\sigma_{A} \sigma_{B}\right)-I \in \Psi^{-1}(G)$. Consequently, the product $\sigma_{A}(x, \xi) \sigma_{B}(x, \xi)$ is an invertible matrix for all sufficiently large $\langle\xi\rangle$ and $\left\|\left(\sigma_{A} \sigma_{B}\right)^{-1}\right\|_{o p} \leq C$. It follows then also that $\sigma_{A}(x, \xi)$ is an invertible matrix for all sufficiently large $\langle\xi\rangle$. From this and the equality $\sigma_{A}^{-1}\left(\sigma_{A} \sigma_{B}\right)=\sigma_{B}$ we obtain

$$
\left\|\sigma_{A}^{-1}\right\|_{o p}=\left\|\sigma_{B}\left(\sigma_{A} \sigma_{B}\right)^{-1}\right\|_{o p} \leq\left\|\sigma_{B}\right\|_{o p}\left\|\left(\sigma_{A} \sigma_{B}\right)^{-1}\right\|_{o p} \leq C\langle\xi\rangle^{-m}
$$

Assume now that $A \in \Psi^{m}(G)$ has the invertible matrix symbol $\sigma_{A}$ satisfying $\left\|\sigma_{A}(x, \xi)^{-1}\right\|_{o p} \leq C\langle\xi\rangle^{-m}$. We can disregard representations $\xi$ with bounded $\langle\xi\rangle$ because they correspond to a smoothing operator. From Theorem 2.2 applied with the difference operators from Lemma 4.4, we get that $\operatorname{Op}\left(\sigma_{A}^{-1}\right) \in \Psi^{-m}(G)$ because we have $\left\|\mathbb{D}^{\gamma} \partial_{x}^{\beta}\left(\sigma_{A}(x, \xi)^{-1}\right)\right\|_{o p} \leq C\langle\xi\rangle^{-m-|\gamma|}$ by Lemma 4.5 with $m_{0}=m, \rho=1$ and $\delta=0$. Since $\mathrm{Op}\left(\sigma_{A}^{-1}\right) \in \Psi^{-m}(G)$, Theorem 10.9.10 in [10] implies that $A$ has a parametrix (given by formula (5)). This implies that $A$ is elliptic. The proof is complete.

\section{Global Hypoellipticity}

We now turn to the analysis of hypoelliptic operators. We recall that an operator $A$ is globally hypoelliptic on $G$ if $A u=f$ and $f \in C^{\infty}(G)$ imply $u \in C^{\infty}(G)$. We use the notation $\mathscr{S}_{\rho, \delta}^{m}(G)$ for the class of symbols for which the corresponding $(\rho, \delta)$-versions of symbol estimates are satisfied, namely, $\sigma_{A} \in \mathscr{S}_{\rho, \delta}^{m}(G)$ if for a strongly admissible selection $\triangle_{1}, \ldots, \triangle_{n} \in \operatorname{diff}^{1}(\widehat{G})$ we have

$$
\left\|\triangle_{\xi}^{\alpha} \partial_{x}^{\beta} \sigma_{A}(x, \xi)\right\|_{o p} \leq C_{\alpha \beta}\langle\xi\rangle^{m-\rho|\alpha|+\delta|\beta|}
$$

for all multi-indices $\alpha, \beta \in \mathbb{N}_{0}^{n}$. Theorem $2.2(B) \Leftrightarrow(C) \Leftrightarrow(D)$ is valid for all $1 \geq \rho>\delta \geq 0$. By $\operatorname{Op}\left(\mathscr{S}_{\rho, \delta}^{m}(G)\right)$ we denote the class of all operators $A$ of the form (2) with symbols $\sigma_{A} \in \mathscr{S}_{\rho, \delta}^{m}(G)$. Such operators can be readily seen to be continuous on $C^{\infty}(G)$. In the previous notation we have $\mathscr{S}^{m}(G)=\mathscr{S}_{1,0}^{m}(G)$, and $\Psi^{m}(G)=\operatorname{Op}\left(\mathscr{S}_{1,0}^{m}(G)\right)$ by Theorem 2.2.

The knowledge of the full symbols allows us to establish an analogue of the wellknown hypoellipticity result of Hörmander [7] on $\mathbb{R}^{n}$, requiring conditions on lower order terms of the symbol. The following theorem is a matrix-valued symbol criterion for (local) hypoellipticity.

Theorem 5.1 Let $m \geq m_{0}$ and $1 \geq \rho>\delta \geq 0$. Let $A \in \mathrm{Op}\left(\mathscr{S}_{\rho, \delta}^{m}(G)\right)$ be a pseudodifferential operator with the matrix-valued symbol $\sigma_{A}=\sigma_{A}(x, \xi) \in \mathscr{S}_{\rho, \delta}^{m}(G)$ which is invertible for all but finitely many $[\xi] \in \widehat{G}$, and for all such $\xi$ satisfies

$$
\left\|\sigma_{A}(x, \xi)^{-1}\right\|_{o p} \leq C\langle\xi\rangle^{-m_{0}}
$$


for all $x \in G$. Assume also that for a strongly admissible collection of difference operators we have

$$
\left\|\sigma_{A}(x, \xi)^{-1}\left[\triangle_{\xi}^{\alpha} \partial_{x}^{\beta} \sigma_{A}(x, \xi)\right]\right\|_{o p} \leq C\langle\xi\rangle^{-\rho|\alpha|+\delta|\beta|}
$$

for all multi-indices $\alpha, \beta$, all $x \in G$, and all but finitely many [ $\xi]$. Then there exists an operator $B \in \operatorname{Op}\left(\mathscr{S}_{\rho, \delta}^{-m_{0}}(G)\right)$ such that $A B-I$ and $B A-I$ belong to $\Psi^{-\infty}(G)$. Consequently, we have

$$
\operatorname{sing} \operatorname{supp} A u=\operatorname{sing} \operatorname{supp} u \text { for all } u \in \mathcal{D}^{\prime}(G) \text {. }
$$

Proof First of all, we can assume at any stage of the proof that (14) and (15) hold for all $[\xi] \in \widehat{G}$ since we can always modify symbols for small $\langle\xi\rangle$ which amounts to adding a smoothing operator. Then we observe that if we apply Theorem 2.2 with difference operators $\mathbb{D}$ from Lemma 4.4, it follows from Lemma 4.5 that $\sigma_{A}^{-1} \in \mathscr{S}_{\rho, \delta}^{-m_{0}}(G)$. Let us show next that $\sigma_{A}^{-1} \triangle_{\xi}^{\alpha} \partial_{x}^{\beta} \sigma_{A} \in \mathscr{S}_{\rho, \delta}^{-\rho|\alpha|+\delta|\beta|}(G)$. Differentiating the equality

$$
\sigma_{A}\left(\sigma_{A}^{-1} \triangle_{\xi}^{\alpha} \partial_{x}^{\beta} \sigma_{A}\right)=\triangle_{\xi}^{\alpha} \partial_{x}^{\beta} \sigma_{A}
$$

we get

$$
\partial_{x_{j}}\left(\sigma_{A}^{-1} \triangle_{\xi}^{\alpha} \partial_{x}^{\beta} \sigma_{A}\right)=\sigma_{A}^{-1} \partial_{x_{j}} \triangle_{\xi}^{\alpha} \partial_{x}^{\beta} \sigma_{A}-\left(\sigma_{A}^{-1} \partial_{x_{j}} \sigma_{A}\right)\left(\sigma_{A}^{-1} \triangle_{\xi}^{\alpha} \partial_{x}^{\beta} \sigma_{A}\right)
$$

From this and (15) it follows that $\left\|\partial_{x_{j}}\left(\sigma_{A}^{-1} \triangle_{\xi}^{\alpha} \partial_{x}^{\beta} \sigma_{A}\right)\right\|_{o p} \leq C\langle\xi\rangle^{-\rho|\alpha|+\delta|\beta|+\delta}$. Continuing this argument, we get that $\left\|\partial_{x}^{\gamma}\left(\sigma_{A}^{-1} \triangle_{\xi}^{\alpha} \partial_{x}^{\beta} \sigma_{A}\right)\right\|_{o p} \leq C\langle\xi\rangle^{-\rho|\alpha|+\delta|\beta+\gamma|}$ for all $\gamma$. For differences, the Leibniz formula in Proposition 4.3 applied to (16) gives

$$
\begin{aligned}
& \mathbb{D}_{i j}\left(\sigma_{A}^{-1} \triangle_{\xi}^{\alpha} \partial_{x}^{\beta} \sigma_{A}\right)+\left(\sigma_{A}^{-1} \mathbb{D}_{i j} \sigma_{A}\right)\left(\sigma_{A}^{-1} \triangle_{\xi}^{\alpha} \partial_{x}^{\beta} \sigma_{A}\right) \\
& \quad+\sum_{k}\left(\sigma_{A}^{-1} \mathbb{D}_{i k} \sigma_{A}\right) \mathbb{D}_{k j}\left(\sigma_{A}^{-1} \triangle_{\xi}^{\alpha} \partial_{x}^{\beta} \sigma_{A}\right)=\sigma_{A}^{-1} \mathbb{D}_{i j} \triangle_{\xi}^{\alpha} \partial_{x}^{\beta} \sigma_{A}
\end{aligned}
$$

Writing these equations for all $i, j$ gives a linear system on $\left\{\mathbb{D}_{i j}\left(\sigma_{A}^{-1} \triangle_{\xi}^{\alpha} \partial_{x}^{\beta} \sigma_{A}\right)\right\}_{i j}$ with coefficients of the form $I+\sigma_{A}^{-1} \mathbb{D}_{k l} \sigma_{A}$ for suitable sets of indices $k$, $l$. Since $\left\|\sigma_{A}^{-1} \mathbb{D}_{k l} \sigma_{A}\right\|_{o p} \leq C\langle\xi\rangle^{-\rho}$ by (15) and Theorem 2.2, we can solve it for matrices $\left\{\mathbb{D}_{i j}\left(\sigma_{A}^{-1} \triangle_{\xi}^{\alpha} \partial_{x}^{\beta} \sigma_{A}\right)\right\}_{i j}$ for large $\langle\xi\rangle$. Finally, since

$$
\begin{gathered}
\left\|\left(\sigma_{A}^{-1} \mathbb{D}_{i j} \sigma_{A}\right)\left(\sigma_{A}^{-1} \triangle_{\xi}^{\alpha} \partial_{x}^{\beta} \sigma_{A}\right)\right\|_{o p} \leq C\langle\xi\rangle^{-\rho|\alpha|+\delta|\beta|-\rho} \\
\left\|\sigma_{A}^{-1} \mathbb{D}_{i j} \triangle_{\xi}^{\alpha} \partial_{x}^{\beta} \sigma_{A}\right\|_{o p} \leq C\langle\xi\rangle^{-\rho|\alpha|+\delta|\beta|-\rho}
\end{gathered}
$$

we get that $\left\|\mathbb{D}_{i j}\left(\sigma_{A}^{-1} \triangle_{\xi}^{\alpha} \partial_{x}^{\beta} \sigma_{A}\right)\right\|_{o p} \leq C\langle\xi\rangle^{-\rho|\alpha|+\delta|\beta|-\rho}$ for all $i, j$. An induction argument similar to the one in the proof of Lemma 4.5 shows that $\sigma_{A}^{-1} \triangle_{\xi}^{\alpha} \partial_{x}^{\beta} \sigma_{A} \in$ 
$\mathscr{S}_{\rho, \delta}^{-\rho|\alpha|+\delta|\beta|}(G)$. Let us now denote $\sigma_{B_{0}}(x, \xi)=\sigma_{A_{0}}(x, \xi)^{-1}$, and let $\sigma_{B} \sim$ $\sum_{N=0}^{\infty} \sigma_{B_{N}}$, where

$$
\sigma_{B_{N}}(x, \xi)=-\sigma_{B_{0}}(x, \xi) \sum_{k=0}^{N-1} \sum_{|\gamma|=N-k} \frac{1}{\gamma !}\left[\Delta_{\xi}^{\gamma} \sigma_{A}(x, \xi)\right] \partial_{x}^{(\gamma)} \sigma_{B_{k}}(x, \xi) .
$$

One can readily check that $B$ is a parametrix of $A$ (this is a special case of [10, Exercise 10.9.12]). We claim that $\sigma_{B_{N}} \in \mathscr{S}_{\rho, \delta}^{-m_{0}-N}(G)$. We already know that $\sigma_{B_{0}} \in \mathscr{S}_{\rho, \delta}^{-m_{0}}(G)$. Inductively, in view of $\sigma_{A}^{-1} \triangle_{\xi}^{\alpha} \partial_{x}^{\beta} \sigma_{A} \in \mathscr{S}_{\rho, \delta}^{-\rho|\alpha|+\delta|\beta|}(G)$, formula (17) implies that the order of $B_{N}$ is $-(\rho-\delta)|\gamma|+\operatorname{order}\left(B_{k}\right)=-(\rho-\delta)|\gamma|-$ $m_{0}-(\rho-\delta) k=-m_{0}-(\rho-\delta) N$. Thus, $B \in \operatorname{Op}\left(\mathscr{S}_{\rho, \delta}^{-m_{0}}(G)\right)$ is a parametrix for $A$.

Finally, the pseudo-locality of the operator $A$ (which can be proved directly from the symbolic calculus) implies that $\operatorname{sing} \operatorname{supp} A u \subset \operatorname{sing} \operatorname{supp} u$. Writing $u=B(A u)-R u$ with $R \in \Psi^{-\infty}(G)$, we get that

$$
\operatorname{sing} \operatorname{supp} u \subset \operatorname{sing} \operatorname{supp}(A u) \bigcup \operatorname{sing} \operatorname{supp}(R u),
$$

so that $\operatorname{sing} \operatorname{supp} A u=\operatorname{sing} \operatorname{supp} u$ because $R u \in C^{\infty}(G)$.

To obtain global hypoellipticity, it is sufficient to show that an operator $A \in \Psi(G)$ has a parametrix $B$ satisfying subelliptic estimates $\|B f\|_{H^{s}} \lesssim\|f\|_{H^{s+m}}$ for some constant $m$ independent of $s \in \mathbb{R}$.

\subsection{Examples}

We will conclude with a collection of examples on the group SU(2) contrasting local and global hypoellipticities. These examples can be also viewed as examples on the 3 -sphere $\mathbb{S}^{3}$, see Example 2.5. Thus, in what follows we denote $G=\operatorname{SU}(2) \cong \mathbb{S}^{3}$. Although the calculations will rely on the representations of $\mathrm{SU}(2)$, whenever $G$ appears in the statements one can substitute it by either $\mathrm{SU}(2)$ or $\mathbb{S}^{3}$. In contrast to [10] we will base considerations here on the four difference operators $\mathbb{D}_{i j}, i, j \in\{1,2\}$, defined in terms of the first non-trivial representation corresponding to $\ell=\frac{1}{2}$. They are related to $\triangle_{\eta}, \eta \in\{0,+,-\}$ (see Example 2.5), via $\Delta_{-}=\mathbb{D}_{12}, \Delta_{+}=\mathbb{D}_{21}$, and $\triangle_{0}=\mathbb{D}_{11}-\mathbb{D}_{22}$, while $\mathbb{D}_{11}+\mathbb{D}_{22} \in \operatorname{diff}^{2}(\widehat{G})$, and satisfy the Leibniz rule (6). Furthermore, the elementary (homogeneous) first order differential operators $\partial_{0}, \partial_{ \pm} \in \Psi^{1}(G)$ and the Laplacian $\mathcal{L}=-\partial_{0}^{2}-\frac{1}{2}\left(\partial_{-} \partial_{+}+\partial_{+} \partial_{-}\right) \in \Psi^{2}(G)$ satisfy relations in Table 1 .

Invariant vector fields corresponding to the basis Pauli matrices in $\mathfrak{s u}(2)$ are expressible as $\mathrm{D}_{1}=-\frac{\mathrm{i}}{2}\left(\partial_{-}+\partial_{+}\right), \mathrm{D}_{2}=\frac{1}{2}\left(\partial_{-}-\partial_{+}\right)$and $\mathrm{D}_{3}=-\mathrm{i} \partial_{0}$. We refer to $[10,12]$ for the details on these operators and for the explicit formulae for their symbols. We also note that the analysis of invariant operators by Coifman and Weiss in [4] can be interpreted in terms of these difference operators as well. 
Table 1 Difference operators

\begin{tabular}{lllll}
\hline & $\sigma_{\partial_{0}}$ & $\sigma_{\partial_{+}}$ & $\sigma_{\partial_{-}}$ & $\sigma_{\mathcal{L}}$ \\
\hline $\mathbb{D}_{11}$ & $\frac{1}{2} \sigma_{I}$ & 0 & 0 & $-\sigma_{\partial_{0}}+\frac{1}{4} \sigma_{I}$ \\
$\mathbb{D}_{12}$ & 0 & $\sigma_{I}$ & 0 & $-\sigma_{\partial_{-}}$ \\
$\mathbb{D}_{21}$ & 0 & 0 & $\sigma_{I}$ & $-\sigma_{\partial_{+}}$ \\
$\mathbb{D}_{22}$ & $-\frac{1}{2} \sigma_{I}$ & 0 & 0 & $\sigma_{\partial_{0}}+\frac{1}{4} \sigma_{I}$ \\
\hline
\end{tabular}

Example 5.2 We consider $\mathrm{D}_{3}+c$ for constants $c \in \mathbb{C}$. The corresponding symbol is given by

$$
\left(\sigma_{\mathrm{D}_{3}+c}\right)_{m n}^{\ell}=[c-\mathrm{i} m] \delta_{m n}^{\ell}
$$

where, following [10], $m, n \in\{-\ell,-\ell+1, \ldots, \ell-1, \ell\}$ run through half-integers or integers, depending on whether $\ell \in \frac{1}{2} \mathbb{N}_{0}$ is a half-integer or an integer, respectively. We also use the notation $\delta_{m n}^{\ell}$ for the Kronecker delta, i.e. $\delta_{m n}^{\ell}=1$ for $m=n$ and $\delta_{m n}^{\ell}=0$ for $m \neq n$. This symbol $\sigma_{\mathrm{D}_{3}+c}$ is invertible for all $\ell$ if (and only if) $c$ is not an imaginary half-integer, ic $\notin \frac{1}{2} \mathbb{Z}$. The inverse satisfies $\left(\mathrm{D}_{3}+c\right)^{-1} \in \mathrm{Op}\left(\mathscr{S}_{0,0}^{0}(G)\right)$, based on

$$
\begin{aligned}
& \mathbb{D}_{11} \sigma_{\left(\mathrm{D}_{3}+c\right)^{-1}}=-\frac{1}{2} \sigma_{\left(\mathrm{D}_{3}+c\right)^{-1}} \sigma_{\left(\mathrm{D}_{3}+c+\frac{1}{2}\right)^{-1}}, \\
& \mathbb{D}_{22} \sigma_{\left(\mathrm{D}_{3}+c\right)^{-1}}=\frac{1}{2} \sigma_{\left(\mathrm{D}_{3}+c\right)^{-1}} \sigma_{\left(\mathrm{D}_{3}+c-\frac{1}{2}\right)^{-1}},
\end{aligned}
$$

and $\mathbb{D}_{12} \sigma_{\left(\mathrm{D}_{3}+c\right)^{-1}}=\mathbb{D}_{21} \sigma_{\left(\mathrm{D}_{3}+c\right)^{-1}}=0$, combined with Leibniz' rule. As a consequence we see that the operator $\mathrm{D}_{3}+c$ satisfies subelliptic estimates with loss of one derivative and are thus globally hypoelliptic.

The statement is sharp: the spectrum of $\mathrm{D}_{3}$ consists of all imaginary half-integers and all eigenspaces are infinite-dimensional (stemming from the fact that each such imaginary half-integer hits infinitely many representations for which $-\ell \leq \mathrm{i} c \leq \ell$ and $\mathrm{i} c+\ell \in \mathbb{Z}$ ). In particular, eigenfunctions can be irregular, e.g., distributions which do not belong to certain negative order Sobolev spaces.

Example 5.3 By conjugation formulae in $[10,12]$ this example can be extended to give examples of more general left-invariant operators. Namely, let $X \in \mathfrak{g}$ be normalised, $\|X\|=\left\|\mathrm{D}_{3}\right\|$ with respect to the Killing norm, and let $\partial_{X}$ be the derivative with respect to the vector field $X$. Then it was shown that the vector field $X$ can be conjugated to $\mathrm{D}_{3}$ so that the operator $\partial_{X}+c$ is globally hypoelliptic if and only if ic $\notin \frac{1}{2} \mathbb{Z}$.

Moreover, by conjugation we can readily see that in a suitably chosen basis in the representation spaces, the symbol of the operator $\partial_{X}^{k}, k \in \mathbb{N}$, is

$$
\left(\sigma_{\partial_{X}^{k}}\right)_{m n}^{\ell}=\left[(-\mathrm{i} m)^{k}\right] \delta_{m n}^{\ell}
$$


Denoting $\left(\frac{1}{2} \mathbb{Z}\right)^{k}=\left\{2^{-k} l^{k}: l \in \mathbb{Z}\right\}$, we see that the operator $\partial_{X}^{k}+c$ is globally hypoelliptic if and only if $c \notin-(-\mathrm{i})^{k}\left(\frac{1}{2} \mathbb{Z}\right)^{k}$. In particular, for $k=2$, the second order differential operator $\partial_{X}^{2}+c$ is globally hypoelliptic if and only if $4 c \notin\left\{l^{2}: l \in \mathbb{Z}\right\}$.

It is also interesting to note that the operators $\partial_{X}^{2}+a(x) \partial_{X}$ are never globally hypoelliptic for all complex-valued functions $a \in C^{\infty}(G)$. Indeed, in a suitably chosen basis of the representation spaces in $\widehat{\mathrm{SU}(2)}$ their symbols are diagonal, and $\left(\sigma_{\partial_{X}^{2}+a(x) \partial_{X}}\right)_{m n}^{\ell}=0$ for $\ell \in \mathbb{N}$ and $m=n=0$. For simplicity in the following construction we assume that $\partial_{X}=\mathrm{D}_{3}$. If we set $\widehat{f}(\ell)_{m n}=1$ for $\ell \in \mathbb{N}$ and $m=n=0$, and to be zero otherwise, we have $\left(\sigma_{\partial_{X}^{2}+a(x) \partial_{X}}\right) \widehat{f}=0$, so that $\left(\partial_{X}^{2}+a(x) \partial_{X}\right) f=0$ by (2). By the Fourier inversion formula we see that $\widehat{f}$ is the Fourier transform of the function

$$
f(x)=\sum_{\ell \in \mathbb{N}_{0}}(2 \ell+1) t_{00}^{\ell}(x)
$$

so that $f \in \mathcal{D}^{\prime}(G)$ is not smooth but $\left(\partial_{X}^{2}+a(x) \partial_{X}\right) f=0$.

We note that operators from Example 5.3 are not covered by Hörmander's sum of the squares theorem [8]. Although the following example of the sub-Laplacian is covered by Hörmander's theorem, Theorem 5.1 provides its inverse as a pseudo-differential operator with the global matrix-valued symbol in the class $\mathscr{S}_{\frac{1}{2}, 0}^{-1}(G)$.

Example 5.4 Sub-Laplacian. The sub-Laplacian on $G$ is defined as $\mathcal{L}_{s}=\mathrm{D}_{1}^{2}+\mathrm{D}_{2}^{2} \in$ $\Psi^{2}(G)$ and has the symbol

$$
\left(\sigma_{\mathcal{L}_{s}}\right)_{m n}^{\ell}=\left[m^{2}-\ell(\ell+1)\right] \delta_{m n}^{\ell} .
$$

This symbol is invertible for all $\ell>0$. It satisfies the hypoellipticity assumption of Theorem 5.1 with $\rho=\frac{1}{2}$. For this we note that $\mathbb{D}_{11} \sigma_{\mathcal{L}_{s}}=\mathbb{D}_{22} \sigma_{\mathcal{L}_{s}}=0$ and $\mathbb{D}_{12} \sigma_{\mathcal{L}_{s}}=-\sigma_{\partial_{-}}, \mathbb{D}_{21} \sigma_{\mathcal{L}_{s}}=-\sigma_{\partial_{+}}$. Estimate (15) is evident for $\alpha=0$ and $|\alpha| \geq 2$. Hence it remains to check that

$$
\left\|\left(\sigma_{\mathcal{L}_{S}}\right)^{-1} \mathbb{D}_{12} \sigma_{\mathcal{L}_{s}}\right\|=\left\|\left(\sigma_{\mathcal{L}_{s}}\right)^{-1} \sigma_{\partial_{-}}\right\| \lesssim \ell^{-1 / 2}
$$

(and the analogous statement for $\mathbb{D}_{21}$ ). This can be done by direct computation,

$$
\left(\sigma_{\mathcal{L}_{s}}^{-1} \mathbb{D}_{12} \sigma_{\mathcal{L}_{s}}\right)_{m n}^{\ell}=\left[\frac{\sqrt{(\ell+n)(\ell-n+1)}}{(n-1)^{2}-\ell(\ell+1)}\right] \delta_{m, n-1}^{\ell},
$$

the entries corresponding to $\ell=-n$ vanish, while all others are bounded by $\sqrt{2 / \ell}$. Hence, $\mathcal{L}_{s}$ has a pseudo-differential parametrix $\mathcal{L}_{s}^{\sharp} \in \operatorname{Op}\left(\mathscr{S}_{\frac{1}{2}, 0}^{-1}(G)\right)$. In particular, from this we automatically get the subelliptic estimate

$$
\|f\|_{H^{s}(G)} \leq C\left\|\mathcal{L}_{s} f\right\|_{H^{s-1}(G)}
$$

for all $s \in \mathbb{R}$. 
Example 5.5 Heat operator. We consider the analogue of the heat operator on SU(2), namely the operator $H=\mathrm{D}_{3}-\mathrm{D}_{1}^{2}-\mathrm{D}_{2}^{2}$. Writing $H=\mathrm{D}_{3}-\mathcal{L}_{s}$, we see that its symbol is

$$
\left(\sigma_{H}\right)_{m n}^{\ell}=\left[-\mathrm{i} m-m^{2}+\ell(\ell+1)\right] \delta_{m n}^{\ell}
$$

Consequently, using the differences for the sub-Laplacian, we see that $\mathbb{D}_{11} \sigma_{H}=$ $-\frac{i}{2} \sigma_{I}, \mathbb{D}_{22} \sigma_{H}=\frac{i}{2} \sigma_{I}, \mathbb{D}_{12} \sigma_{H}=-\mathbb{D}_{12} \sigma_{\mathcal{L}_{s}}=\sigma_{\partial_{-}}, \mathbb{D}_{21} \sigma_{H}=-\mathbb{D}_{21} \sigma_{\mathcal{L}_{s}}=\sigma_{\partial_{+}}$. By an argument similar to that for the sub-Laplacian, the operator $H$ has a parametrix $H^{\sharp} \in \mathrm{Op}\left(\mathscr{S}_{\frac{1}{2}, 0}^{-1}(G)\right)$ and, in particular, we have

$$
\|f\|_{H^{s}(G)} \leq C\|H f\|_{H^{s-1}(G)}
$$

for all $s \in \mathbb{R}$.

Example 5.6 Schrödinger operator. We consider the analogue of the Schrödinger operator on $G$, namely the operators $S_{ \pm}= \pm \mathrm{iD}_{3}-\mathrm{D}_{1}^{2}-\mathrm{D}_{2}^{2}$. We will treat both \pm cases simultaneously by keeping the sign \pm or $\mp$. Writing $S_{ \pm}= \pm \mathrm{iD}_{3}-\mathcal{L}_{s}$, we see that their symbols are

$$
\left(\sigma_{S_{ \pm}}\right)_{m n}^{\ell}=\left[ \pm m-m^{2}+\ell(\ell+1)\right] \delta_{m n}^{\ell}
$$

Consequently, we see that these symbols are not invertible. Analogously to Example 5.3 we can construct distributions in the null spaces of $S_{ \pm}$. Namely, if we define $\widehat{f_{ \pm}}(\ell)_{m n}=1$ for $m=n=\mp \ell$, and to be zero otherwise, then we readily see that the functions $f_{ \pm}(x)=\sum_{\ell \in \frac{1}{2} \mathbb{N}_{0}}(2 \ell+1) t_{\mp \ell \mp \ell}^{\ell}(x)$ satisfy $S_{ \pm} f_{ \pm}=0$, but $f_{ \pm} \in \mathcal{D}^{\prime}(G)$ are not smooth.

On the other hand, the operators $S_{ \pm}+c$ become globally hypoelliptic for almost all constants $c$. Let us consider the case $S_{-}$, the argument for $S_{+}$is similar. Since the symbol of $S_{-}+c$ is

$$
\left(\sigma_{S_{-}+c}\right)_{m n}^{\ell}=\left[c-m-m^{2}+\ell(\ell+1)\right] \delta_{m n}^{\ell}
$$

we see that it is invertible if and only if $c \notin \mathbb{N}_{0}$. If $c \in \mathbb{C} \backslash \mathbb{N}_{0}$ then $\left\|\sigma_{\left(S_{-}+c\right)^{-1}}\right\|_{o p} \leq C$, and hence $S_{-}+c$ is globally hypoelliptic.

Example 5.7 D'Alembertian. We consider $W=\mathrm{D}_{3}^{2}-\mathrm{D}_{1}^{2}-\mathrm{D}_{2}^{2}$. Writing $W=\mathrm{D}_{3}^{2}-$ $\mathcal{L}_{s}=2 \mathrm{D}_{3}^{2}-\mathcal{L}$ with the Laplace operator $\mathcal{L}=\mathrm{D}_{1}^{2}+\mathrm{D}_{2}^{2}+\mathrm{D}_{3}^{2}$, we see that the symbol of $W \in \Psi^{2}(G)$ is given by

$$
\left(\sigma_{W}\right)_{m n}^{\ell}=\left[-2 m^{2}+\ell(\ell+1)\right] \delta_{m n}^{\ell}
$$

If $\ell \in \frac{1}{2} \mathbb{N}_{0}$ is a half-integer, we easily see that $\left|-2 m^{2}+\ell(\ell+1)\right| \geq \frac{1}{4}$ for all $\ell-m \in \mathbb{Z},|m| \leq \ell$. However, if $\ell$ is an integer, the triangular numbers $\frac{\ell(\ell+1)}{2}$ 
are squares if $\ell=\ell_{k}=\left\lfloor(3+2 \sqrt{2})^{k} / 4\right\rfloor, k \in \mathbb{N}$, see [19, A001109]. Denoting by $m_{k}=\sqrt{\ell_{k}\left(\ell_{k}+1\right) / 2}$, we see that the function

$$
f(x)=\sum_{k=1}^{\infty}\left(2 \ell_{k}+1\right) t_{m_{k} m_{k}}^{\ell_{k}}(x)
$$

satisfies $W f=0$ but $f \in \mathcal{D}^{\prime}(G)$ is not smooth.

Since the only non-zero triangular number which is also a cube is 1 (see, e.g., [22]) we see that the operator $2 \mathrm{iD}_{3}^{3}-\mathcal{L}$ is globally hypoelliptic.

Example 5.8 We consider $P=\mathrm{D}_{1}^{2}-\mathrm{D}_{2}^{2}=-\frac{1}{2}\left(\partial_{-}^{2}+\partial_{+}^{2}\right)$. The corresponding symbol satisfies

$$
\begin{aligned}
2\left(\sigma_{P}\right)_{m n}^{\ell}= & \sqrt{(\ell+n)(\ell+n-1)(\ell-n+2)(\ell-n+1)} \delta_{m, n-2}^{\ell} \\
& +\sqrt{(\ell+m)(\ell+m-1)(\ell-m+2)(\ell-m+1)} \delta_{m-2, n}^{\ell} .
\end{aligned}
$$

Furthermore, by Leibniz' rule we deduce $\mathbb{D}_{11} \sigma_{P}=\mathbb{D}_{22} \sigma_{P}=0$ and $\mathbb{D}_{12} \sigma_{P}=-\sigma_{\partial_{+}}$, $\mathbb{D}_{21} \sigma_{P}=-\sigma_{\partial_{-}}$. The symbol $\sigma_{P}$ is invertible only for $\ell+\frac{1}{2} \in 2 \mathbb{N}_{0}$. Therefore, $P$ is neither globally hypoelliptic nor does it satisfy subelliptic estimates.

\section{References}

1. Agranovich, M.S.: Elliptic pseudodifferential operators on a closed curve. (Russian). Trudy Mosk. Mat. Obshch. 47, 22-67 (1984)

2. Beals, R.: Characterization of pseudo-differential operators and applications. Duke Math. J. 44, 45-57 (1977)

3. Bergamasco, A., Zani, S., Luís, S.: Prescribing analytic singularities for solutions of a class of vector fields on the torus. Trans. Am. Math. Soc. 357, 4159-4174 (2005)

4. Coifman, R., Weiss, G.: Analyse Harmonique Non-Commutative sur Certains Espaces Homogènes. Lecture Notes in Mathematics. Springer, Berlin (1971)

5. Dasgupta, A., Ruzhansky, M.: Gohberg lemma, compactness, and essential spectrum of operators on compact Lie groups. to appear in, J. Anal. Math. arXiv:1306.0041

6. Himonas, A., Petronilho, G.: Global hypoellipticity and simultaneous approximability. J. Funct. Anal. 170, 356-365 (2000)

7. Hörmander, L.: Pseudo-differential operators and hypoelliptic equations. In: Proceeding of symposia pure mathematics, pp. 138-183. Amer. Math. Soc., 1967

8. Hörmander, L.: Hypoelliptic second order differential equations. Acta Math. 119, 147-171 (1967)

9. Kohn, J.J., Nirenber, L.: An algebra of pseudo-differential operators. Comm. Pure Appl. Math. 18, 269-305 (1965)

10. Ruzhansky, M., Turunen, V.: Pseudo-Differential Operators and Symmetries. Birkhäuser, Basel (2010)

11. Ruzhansky, M., Turunen, V.: Quantization of pseudo-differential operators on the torus. J. Fourier Anal. Appl. 16, 943-982 (2010)

12. Ruzhansky, M., Turunen, V.: Global quantization of pseudo-differential operators on compact Lie groups, SU(2) and 3-sphere. Int. Math. Res. Notices IMRN 11, 2439-2496 (2013). doi:10.1093/imrn/ rns 122

13. Ruzhansky, M., Turunen, V.: Sharp Gårding inequality on compact Lie groups. J. Funct. Anal. 260, 2881-2901 (2011)

14. Ruzhansky, M., Wirth, J.: On multipliers on compact Lie groups. Funct. Anal. Appl. 47, 72-75 (2013)

15. Ruzhansky, M., Wirth, J.: $L^{p}$ Fourier multipliers on compact Lie groups. arXiv:1102.3988v1.

16. Sherman, T.: Fourier analysis on the sphere. Trans. Am. Math. Soc. 209, 1-31 (1975) 
17. Sherman, T.: The Helgason Fourier transform for compact Riemannian symmetric spaces of rank one. Acta Math. 164, 73-144 (1990)

18. Shubin, M.A.: Pseudodifferential Operators and Spectral Theory. Springer, Berlin (1987)

19. Sloane, N.J.A. (ed.): The On-Line Encyclopedia of Integer Sequences. Published electronically at www.research.att.com/ njas/sequences/. Sequence A001108

20. Taylor, M.E.: Noncommutative microlocal analysis. I. Mem. Amer. Math. Soc. 52(313) (1984)

21. Taylor, M.E.: Beals-Cordes -type characterizations of pseudodifferential operators. Proc. Am. Math. Soc. 125, 1711-1716 (1997)

22. Weisstein, E.W.: Cubic Triangular Number, from MathWorld-A Wolfram Web Resource. http:// mathworld.wolfram.com/CubicTriangularNumber.html

23. Widom, H.: A complete symbolic calculus for pseudodifferential operators. Bull. Sci. Math. 104, 19-63 (1980) 